\title{
Implicit Active Contours for Automatic Brachytherapy Seed Segmentation in Fluoroscopy
}

\author{
Eric Moult,${ }^{a}$ Clif Burdette, ${ }^{b}$ Danny Song,${ }^{c}$ Gabor Fichtinger, ${ }^{a}$ and Pascal Fallavollita ${ }^{d}$ \\ ${ }^{a}$ Queen's University, Kingston, Ontario, Canada; \\ ${ }^{b}$ Acoustic MedSystems, Inc., Champaign, Illinois, USA; \\ ${ }^{c}$ Johns Hopkins Hospital, Baltimore, Maryland, USA; \\ ${ }^{d}$ Technische Universität München, Munich, Germany
}

\begin{abstract}
Motivation: In prostate brachytherapy, intra-operative dosimetry would be ideal to allow for rapid evaluation of the implant quality while the patient is still in the treatment position. Such a mechanism, however, requires 3-D visualization of the currently deposited seeds relative to the prostate. Thus, accurate, robust, and fully-automatic seed segmentation is of critical importance in achieving intra-operative dosimetry. Methodology: Implanted brachytherapy seeds are segmented by utilizing a region-based implicit active contour approach. Overlapping seed clusters are then resolved using a simple yet effective declustering technique. Results: Ground-truth seed coordinates were obtained via a published segmentation technique. A total of 248 clinical C-arm images from 16 patients were used to validate the proposed algorithm resulting in a $98.4 \%$ automatic detection rate with a corresponding $2.5 \%$ false-positive rate. The overall mean centroid error between the ground-truth and automatic segmentations was measured to be 0.42 pixels, while the mean centroid error for overlapping seed clusters alone was measured to be 0.67 pixels. Conclusion: Based on clinical data evaluation and validation, robust, accurate, and fully-automatic brachytherapy seed segmentation can be achieved through the implicit active contour framework and subsequent seed declustering method.
\end{abstract}

Keywords: Prostate brachytherapy, Intra-operative dosimetry, Active contours, Segmentation, Seed declustering

\section{INTRODUCTION}

\subsection{Clinical Motivation}

With an estimated 217,730 new cases in 2010 alone, prostate cancer is the most common cancer amongst American men, accounting for nearly $28 \%$ of all male cancers; it is also the second leading cause of cancerrelated death among American men. ${ }^{1}$ Brachytherapy, a definitive treatment option for early stage prostate cancer, demonstrates excellent long-term disease-free survival and is chosen by over 60,000 men annually. The brachytherapy procedure entails permanent implantation of small radioactive isotope capsules, commonly called seeds, into the prostate to eliminate the cancer via radiation. The success of the procedure hinges on achieving the precise implant placement necessary for the required dose distribution. Intra-operative dosimetry would enable the physician to account for deviations from the initial seed placement plan and tailor the remaining dose so as to eradicate the cancer while minimizing harm to the surrounding healthy tissues. ${ }^{2}$

A critical step on the path to intra-operative dosimetry is achieving accurate, robust, and fully-automatic seed segmentation. The task of prostate seed segmentation is complicated by the fact that C-arm X-ray images generally have low signal-to-noise ratios and are populated with background structures of varying intensity; ${ }^{3}$ hence, simple approaches, such as straightforward thresholding, yield poor results. ${ }^{4}$ Furthermore, because many images contain seeds which lay on top of one another, or overlap, techniques to resolve these clusters into their constituent components are required.

Further author information: (Send correspondence to P. Fallavollita)

P. Fallavollita: E-mail: fallavol@in.tum.de 


\subsection{Prior Work in Brachytherapy Implant Segmentation}

There have been several prior investigations into prostate seed segmentation. ${ }^{3-5}$ In Lam et al. ${ }^{3}$ a spoke transform was developed to facilitate seed segmentation; however, operator intervention was required in selecting the final threshold value. In Tubic et al. ${ }^{4}$ the morphological top-hat transform was used to normalize image illumination, preparing the image for subsequent thresholding which was achieved through the bidimensional entropy method. Next, overlapping seeds were identified using area, width, and length statistics of the clusters and subsequently declustered using an algorithm based on simulated annealing. In the most recent state of the art method, ${ }^{5}$ a tophat by reconstruction algorithm followed by thresholding via Otsu's method was employed. Here, overlapping seeds were identified using intensity information and subsequently separated via k-means clustering.

\subsection{Active Contours}

Active contour models have been successfully applied to image segmentation in a wide variety of applications. The central idea of active contours is to evolve an initial contour, $\mathcal{C}$, based on internal and external energies. ${ }^{6}$ Active contour models can be broadly categorized into edge-based models and region-based models. ${ }^{7}$ Edge-based models typically rely on an edge indicator function, which in turn depends on the image gradient. Edge-based models are formulated so that as the modulus of the image gradient grows, indicating an edge, the propagation of the contour is retarded. In general, models relying on the image gradient are prone to fail when image boundaries are smooth or discontinuous. ${ }^{8}$ In contrast, region-based models do not rely on the image gradient and consequently exhibit improved performance compared to edge-based models when working images characterized by weak or discontinuous boundaries.

Both edge-based and region-based active contours can be formulated in explicit or implicit frameworks. ${ }^{9}$ In the explicit parametric framework there is an inherent difficulty in addressing topological changes - such as splitting and merging of the contour. Implicit active contours overcome this problem as they are developed in the level set formulation, ${ }^{10}$ where the contour, $\mathcal{C}$, is defined as the zero level set of a higher dimensional function, $\phi$, called the level set function.

\section{METHODOLOGY}

We divide the segmentation process into two stages. In the first stage, active contours are employed to segment the implanted seeds, and in the second stage, any overlapping seed clusters are resolved into their constituent components.

\subsection{Region scalable implicit active contours}

To segment the brachytherapy implants, we utilize the region-based implicit active contour model by Li et al.; ${ }^{7}$ this model was selected as it allows for intensity inhomogeneity as well as elimination of the re-initialization process. Consider a 2-D gray-scale image $I: \Omega_{I} \rightarrow \mathbb{R}$, where $\Omega_{I}$ is the image domain. The energy functional proposed by Li et al. ${ }^{7}$ is defined as:

$$
\begin{aligned}
\mathcal{E}\left(\phi, f_{1}, f_{2}\right)= & \sum_{i=1}^{2} \lambda_{i} \int_{\Omega_{I}}\left(\int_{\Omega_{I}} K_{\sigma}(\mathbf{x}-\mathbf{y})\left|I(\mathbf{y})-f_{i}(\mathbf{x})\right|^{2} M_{i}(\phi(\mathbf{y})) d \mathbf{y}\right) d \mathbf{x} \\
& +\nu \int_{\Omega_{I}}|\nabla H(\phi(\mathbf{x}))| d \mathbf{x}+\mu \int_{\Omega_{I}} \frac{1}{2}(|\nabla \phi(\mathbf{x})|-1)^{2} d \mathbf{x}
\end{aligned}
$$

Where $\lambda_{1}$ and $\lambda_{2}$ are positive constants. $K_{\sigma}$ is a Gaussian kernel given by:

$$
K_{\sigma}(\mathbf{u})=\frac{1}{(2 \pi)^{n / 2} \sigma^{n}} e^{\frac{-|\mathbf{u}|^{2}}{2 \sigma^{2}}}
$$


where the parameter $\sigma$ is restricted to positive values. $H(\phi)$ is the Heaviside function, $M_{1}(\phi)=H(\phi)$ and $M_{2}(\phi)=1-H(\phi)$. The functions $f_{1}(\mathbf{x})$ and $f_{2}(\mathbf{x})$ approximate the image intensities outside and inside the contour, respectively. The region-scalability of the energy functional arises because as $\mathbf{x}$ begins to separate from $\mathbf{y}$, the weighting term $K_{\sigma}(\mathbf{x}-\mathbf{y})$ quickly decays thereby reducing the influence of $\left|I(\mathbf{y})-f_{i}(\mathbf{x})\right|^{2}$.

\subsection{Initial Segmentation Phase}

The initial segmentation can be summarized by the following four steps:

STEP 1 filters the original X-ray, producing the processed image on which the active contour will be evolved. For this step, the three filters employed are: a Wiener lowpass filter, ${ }^{11}$ a homomorphic filter, ${ }^{12}$ and a top-hat filter with disk structuring element.

STEP 2 produces the image that is used to initialize the active contour, eliminating the need for a manually defined region of interest (ROI). To form this initialization image, the original X-ray is blurred with a Gaussian filter and then the Kirsch edge filter ${ }^{13}$ is employed. Defining the initial contour as an edge image, as opposed to an arbitrary initialization, has the advantage that the number of iterations evolving $\phi$ can be significantly decreased. Furthermore, the likelihood of segmenting non-seed objects is reduced (see Figure 1; top-right).

STEP 3 evolves the level set function for 80 iterations, after which a binary image is obtained containing the seeds and possibly some lingering noise. For all trials, the energy functional parameters of (1) were set at: $\lambda_{1}=\lambda_{2}=2.8, \nu=0.1 \times 255 \times 255, \mu=1$, and $\sigma=1.5$ (see Figure 1 ; bottom-left).

STEP 4 eliminates any remaining noise in the image. First, very small and very large foreground regionsthat could not correspond to valid seed regions - are removed. Next, foreground regions having an area or length greater than four times the mean statistics are removed. Finally, the remaining regions are grown radially using a disc structuring element, and only foreground regions corresponding to the largest grown cluster are kept (see Figure 1; bottom-right).

\subsection{Overlapping Seed Declustering}

As there will be certain pixel groupings in the segmented image that correspond to two or more clustered seeds, seed declustering techniques to resolve such groupings are required. To this end, we first describe a method to decluster a general $N$ seed cluster, and then address the issue of how to address such seed clusters.

\subsubsection{Particle Based Separation}

The general idea of our particle based declustering technique is to treat each identified cluster as an empty container and simulate how charged particles would separate if they were released into this container. In particular, given a cluster made up of $N$ different seeds, we define $N$ different types of particles, where similarly typed particles are attracted to one another and dissimilarly typed particles are repulsed by one another. We formulate the particle based declustering technique in two steps: first, $N$ differently typed particles are introduced into the container and their equilibrium position is found, and second, subsequent particles are introduced into the container - one-by-one and of alternating charge - until the container is full. This two step method can be described as:

STEP 1 arranges the first $N$ particles. Let $\mathcal{C}$ be the set of pixel locations in the seed cluster to be resolved; here we assume $N \leq|\mathcal{C}|$, where $|\mathcal{C}|$ is the number of pixels in $\mathcal{C}$ (in practice, we have $N<<|\mathcal{C}|$ ). Also, let $\left(p_{0}, p_{1}, \ldots, p_{N-1}\right)$ be the first $N$ seeds, where $p_{i}=\left(a_{i}, c_{i}\right)$. Here, $p_{i}(1)=a_{i}=\left(x_{i}, y_{i}\right)$ is the position of particle $p_{i}$, and $p_{i}(2)=c_{i} \in\left\{T^{0}, T^{1}, \ldots, T^{N-1}\right\}$ is the charge of particle $p_{i}$. We specify the charges of these first $N$ particles to be $p_{i}(2)=T^{i}$, and the positions to be:

$$
\left(p_{0}(1), p_{1}(1), \ldots, p_{N-1}(1)\right)=\min _{\left(\ell_{0}, \ell_{1}, \ldots, \ell_{N-1}\right)} \sum_{(j, k) \in \mathcal{B}} \frac{1}{\left\|\ell_{j}-\ell_{k}\right\|}
$$


where $\ell_{i}=\left(x_{i}, y_{i}\right) \in \mathcal{C}$ is a pixel location in the cluster; we specify that $\ell_{0} \neq \ell_{1} \neq \ldots \neq \ell_{N-1}$. We also have that $\mathcal{B}=\{(j, k) \mid j, k \in\{0,1, \ldots, N-1\}, j<k\}$, and $\left\|\ell_{i}\right\|=\left(x_{i}^{2}+y_{i}^{2}\right)^{\frac{1}{2}}$. For $N>1$, there will be multiple $\left(\ell_{0}, \ell_{1}, \ldots, \ell_{N-1}\right)$ that minimize $(3)$; one of these solutions is selected arbitrarily. We then initialize an assigned particle set $\mathcal{C}_{N-1}^{p}=\bigcup_{i=0}^{N-1}\left\{p_{i}\right\}$, and an assigned position set $\mathcal{C}_{N-1}^{a}=\bigcup_{i=0}^{N-1}\left\{a_{i}\right\}$.

STEP 2 fills the unassigned portion of the cluster with particles. For $N-1<i<|\mathcal{C}|$, particle $p_{i}=\left(a_{i}, c_{i}\right)$, has a charge given by $p_{i}(2)=T^{\bmod _{N}(i)}$, and a position given by:

$$
p_{i}(1)=\min _{a_{k} \in \mathcal{C} \backslash \mathcal{C}_{i-1}^{a}} \sum_{p_{j} \in \mathcal{C}_{i-1}^{p}} \frac{f\left(p_{i}(2), p_{j}(2)\right)}{\left\|a_{k}-p_{j}(1)\right\|}
$$

where,

$$
f\left(c_{i}, c_{j}\right)= \begin{cases}-1 & , c_{i}=c_{j} \\ 1 & , \text { else }\end{cases}
$$

The assigned particle set $\mathcal{C}_{i}^{p}$ and the assigned position set $\mathcal{C}_{i}^{a}$ are given by $\mathcal{C}_{i}^{p}=\mathcal{C}_{i-1}^{p} \cup\left\{p_{i}\right\}$ and $\mathcal{C}_{i}^{a}=$ $\mathcal{C}_{i-1}^{a} \cup\left\{a_{i}\right\}$, respectively. Once the cluster has been filled with particles, the centroid, $\gamma$, of each of the $N$ seeds is calculated as:

$$
\gamma_{i}=\frac{1}{\sum_{p_{j} \in \mathcal{C}_{|\mathcal{C}|-1}^{p}} \delta\left(p_{j}(2), T^{i}\right)} \sum_{p_{j} \in \mathcal{C}_{|\mathcal{C}|-1}^{p}} p_{j}(1) \delta\left(p_{j}(2), T^{i}\right)
$$

where,

$$
\delta\left(c_{j}, T^{i}\right)= \begin{cases}1 & , c_{j}=T^{i} \\ 0 & , \text { else }\end{cases}
$$

\subsubsection{Cluster Detection}

Although we have provided a framework to decluster groups of an arbitrary number of overlapping seeds, in practice, cases where $N>3$ are uncommon. Thus, in this work we only consider detecting two and three seed clusters.

Detecting Two Seed Clusters: A pixel group is interpreted as a seed cluster of two seeds if it satisfies either of the following requirements: (1) the length of the pixel group is greater than $1 \frac{1}{2}$ times the mean length and less than 3 times the mean length, or (2) the area of the pixel group is greater than $1 \frac{1}{2}$ times the mean area and less than 3 times the mean area.

Detecting Three Seed Clusters: A pixel group is interpreted as a seed cluster of three seeds if it satisfies either of the following requirements: (1) the length of the pixel group is greater than 3 times the mean length and less than 4 times the mean length, or (2) the area of the pixel group is greater than 3 times the mean area and less than 4 times the mean area. 

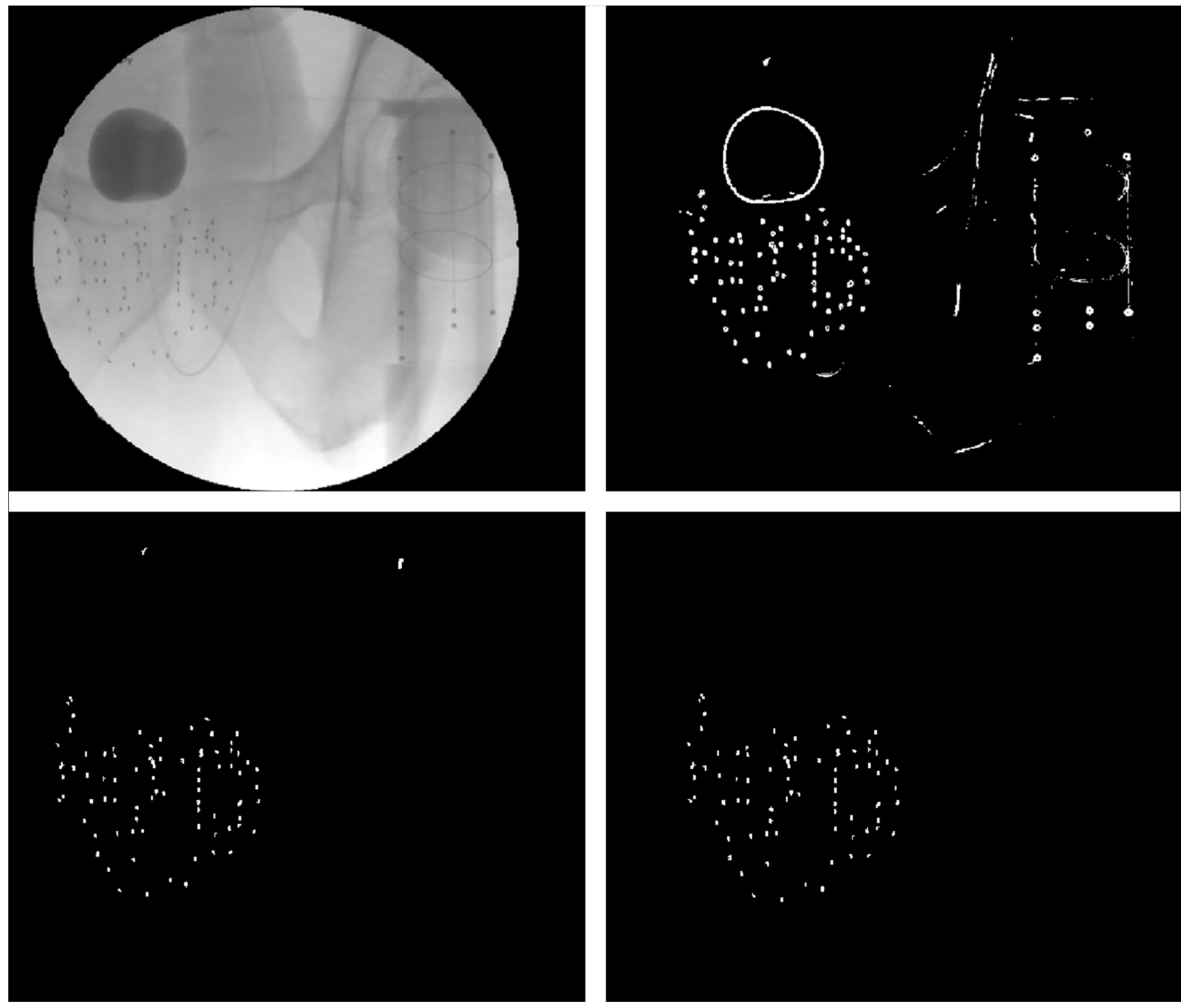

Figure 1. (top-left) Original C-arm X-ray, (top-right) Kirsch filtered X-ray used to initialize active contour model, (bottomleft) after active contour model evolution, and (bottom-right) after noise removal filtering.

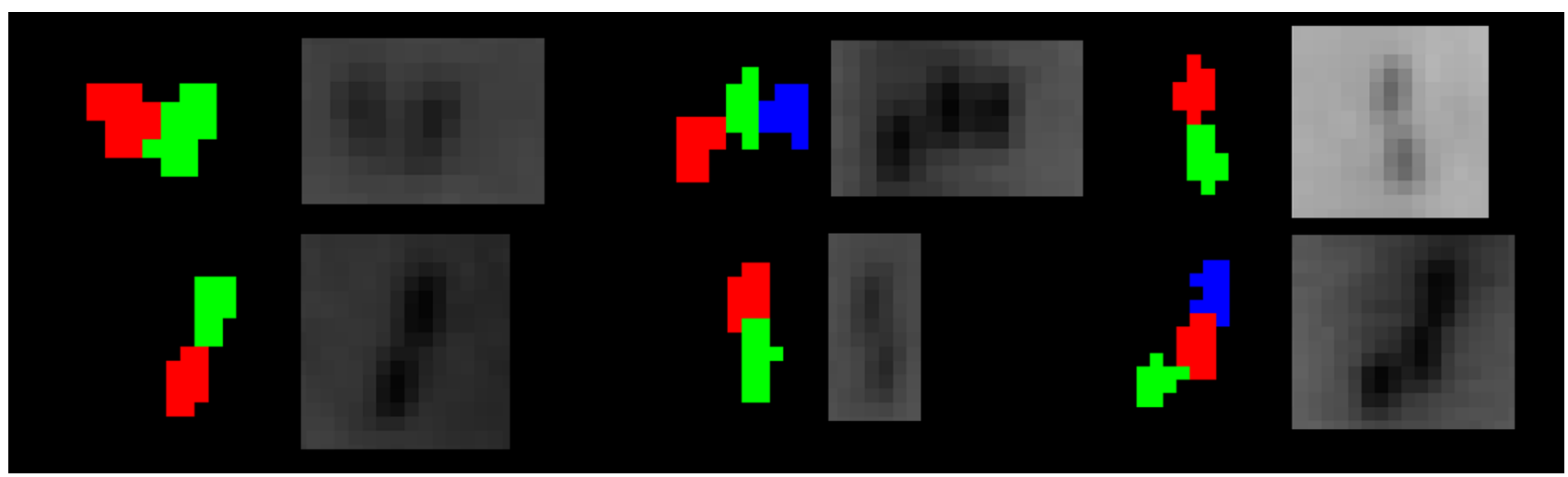

Figure 2. The colored images on the left are the declustered seeds, and the grayscale images on the right are the corresponding overlapping seed clusters from the original C-arm X-ray. 


\section{RESULTS AND DISCUSSION}

We validated the proposed segmentation and declustering method on 248 clinical images from 16 patients. The ground-truth seed coordinates were obtained via manual segmentation. A total of 15354 seeds were manually segmented while 15107 seeds were correctly automatically segmented, resulting in a $98.4 \%$ automatic detection rate. The overall mean centroid error between the ground-truth and automatic segmentations was 0.42 pixels. For the 1383 automatically identified overlapping seeds, the average centroid error between the ground-truth and automatic segmentations was found to be 0.67 pixels. Due to the software used to facilitate the manual segmentation, ground-truth seeds could only be placed at the centers of the image pixels. Since the distance from the center of a pixel to one of its corner is $\sqrt{0.5^{2}+0.5^{2}}=\sqrt{0.5}$, there is $\sqrt{0.5}$ pixel uncertainty associated with the ground-truth segmentations. Finally, 381 seeds were erroneously segmented, leading to a $2.5 \%$ false positive rate. The algorithm was prototyped in a MATLAB/C ++ combination, with an approximate 30 second per-image runtime on a Core 2, $2.4 \mathrm{GHz}$ computer.

The automatic detection rate and overall mean centroid error both attest to the efficacy of the active contour approach to seed segmentation. More subtle are the advantages that the active contour formulation poses over the simpler and faster thresholding based approaches. ${ }^{4,5}$ These advantages arise because even after pre-processing equalization, the X-ray image is still characterized by non-uniform illumination, and thus the thresholding operation can result in pixels either being added onto, or removed from, the detected seeds. Due to the relatively small seed size, even small pixel additions or subtractions can significantly affect the calculated seed centroid. Active contours, and in particular the region scalable formulation that was employed, have the advantage that

they are more adaptable to such non-uniform illumination. Finally, the efficacy of our proposed seed declustering algorithm is apparent from the closeness of the mean centroid error between the clustered and non-clustered seeds.

\section{CONCLUSION}

In this work we have presented a practical technique to robustly segment prostate brachytherapy implants, thereby making an important contribution to both research and clinical practice.

\section{ACKNOWLEDGMENTS}

Eric Moult was supported by the NSERC USRA program and Gabor Fichtinger was supported as a Cancer Care Ontario Research Chair.

\section{REFERENCES}

[1] Jemal, A., Bray, F., Center, M. M., Ferlay, J., Ward, E., and Forman, D., "Global cancer statistics," CA: A Cancer Journal for Clinicians 61(2), 69-90 (2011).

[2] Nag, S., Ciezki, J. P., Cormack, R., Doggett, S., DeWyngaert, K., Edmundson, G. K., Stock, R. G., Stone, N. N., Yu, Y., and Zelefsky, M., "Intraoperative planning and evaluation of permanent prostate brachytherapy: report of the american brachytherapy society," International Journal of Radiation Oncology*Biology*Physics 51(5), 1422 (2001).

[3] Lam, S., Marks, R. J., and Cho, P. S., "Prostate brachytherapy seed segmentation using spoke transform," in $[S P I E], \mathbf{4 3 2 2}(1), 1490-1500$ (2001).

[4] Tubic, D., Zaccarin, A., Beaulieu, L., and Pouliot, J., "Automated seed detection and three-dimensional reconstruction. i. seed localization from fluoroscopic images or radiographs," Medical Physics 28(11), 22722279 (2001).

[5] Kuo, N., Deguet, A., Song, D. Y., Burdette, E. C., Prince, J. L., and Lee, J., "Automatic segmentation of radiographic fiducial and seeds from X-ray images in prostate brachytherapy," Medical Engineering 83 Physics (2011). (in press).

[6] Weeratunga, S. K. and Kamath, C., "Investigation of implicit active contours for scientific image segmentation," in [VCIP], 5308, 210-221 (2004).

[7] Li, C., Kao, C., Gore, J. C., and Ding, Z., "Minimization of region-scalable fitting energy for image segmentation," IEEE Transactions on Image Processing 17, 1940-1949 (October 2008). 
[8] Chan, T. F. and Vese, L. A., "Active contours without edges," IEEE Transactions on Image Processing 10(2), $266-277$ (2001).

[9] Li, C., Xu, C., Gui, C., and Fox, M. D., "Level set evolution without re-initialization: A new variational formulation," in $[C V P R], \mathbf{1}, 430-436$ (2005).

[10] Osher, S. and Sethian, J. A., "Fronts propagating with curvature dependent speed: Algorithms based on Hamilton-Jacobi formulations," Journal of Computational Physics 79(1), 12-49 (1988).

[11] Lim, J. S., [Two-dimensional signal and image processing], Prentice-Hall, Inc., Upper Saddle River, NJ, USA (1990).

[12] Gonzalez, R. C. and Woods, R. E., [Digital Image Processing], Addison-Wesley Longman Publishing Co., Inc., Boston, MA, USA, 2nd ed. (2001).

[13] Kirsch, R. A., "Computer determination of the constituent structure of biological images," in [Computers and Biomedical Research], 4, 315-328 (1971). 